\title{
Dipolar modes in luminous red giants
}

\author{
W. A. Dziembowski \\ Warsaw University Observatory, Aleje Ujazdowskie 4, 00-478, and Copernicus Astronomical Center, ul. Bartycka 18, \\ 00-787 Warsaw, Poland \\ e-mail: wd@astrouw.edu.pl
}

Received 20 July 2011 / Accepted 6 January 2012

\begin{abstract}
Context. Lots of information on solar-like oscillations in red giants has been obtained thanks to observations with CoRoT and Kepler space telescopes. Data on dipolar modes appear most interesting.

Aims. We study properties of dipolar oscillations in luminous red giants to explain mechanism of mode trapping in the convective envelope and to assess what may be learned from the new data.

Methods. Equations for adiabatic oscillations are solved by numerical integration down to the bottom of convective envelope, where the boundary condition is applied. The condition is based on asymptotic decomposition of the fourth order system into components describing a running wave and a nearly uniform shift of radiative core.

Results. If the luminosity of a red giant is sufficiently high $\left(L \gtrsim 100 L_{\odot}\right.$ at $\left.M=2 M_{\odot}\right)$, the dipolar modes become effectively trapped in the acoustic cavity, which covers the outer part of convective envelope. Energy loss caused by gravity wave emission at the envelope base is a secondary or negligible source of damping. Frequencies are insensitive to structure of the deep interior.
\end{abstract}

Key words. stars: oscillations - methods: analytical - stars: interiors - diffusion

\section{Introduction}

Observations with the space-borne telescopes CoRoT and Kepler brought wealth of information about solar-like oscillations in stars along the red giants branch (RGB, Mosser et al. 2011; Hekker et al. 2011, and references therein). In his recent review, Christensen-Dalsgaard (2011) describes these objects as "the most interesting areas of space asteroseismology". Here we focus on dipolar modes in these stars not only because such modes are most important for stellar, in particular red giants (see Montalbán et al. 2011), diagnostic but also because they present some interesting problems for stellar pulsation theory.

What distinguishes dipolar oscillations from those of higher degrees is that the Cowling approximation is not applicable. This approximation has been the basis of standard mode classification and analysis of wave propagation. The problem of a unique dipolar mode classification was solved by Takata $(2005,2006 \mathrm{~b})$. The aspect of our interest in this paper is the effect of the wave trapping in the acoustic cavity which is essential for understanding oscillations observed in red giants.

The gross picture of red giant oscillation emerging from analysis of massive data on red giant oscillations (Mosser et al. 2010) turns out rather simple. The power spectra look similar to that of the Sun from spatially unresolved measurements. The sequences of low degree modes, up to $\ell=3$, are very clearly seen. Only after a closer look a difference in dipolar mode pattern may be noticed. This difference is important and interesting.

Observations largely confirmed what has been predicted by theoretical studies of Dupret et al. (2009) and Montalbán et al. (2010). The first paper was devoted to stochastic excitation of oscillation in red giants and gives values of expected amplitudes and lifetimes for five selected red giants models. The second paper presented an extensive survey of the trapped mode frequencies in red giant models. In very dense eigenfrequency spectra, the modes were identified as the ones corresponding to local minima of inertia. Such choice of the modes was motivated by the results of the first paper and two earlier papers (Dziembowski et al. 2001; Christensen-Dalsgaard 2004), where importance of the mode trapping phenomenon in red giant oscillations was stressed. The goal of the present work is to improve understanding of this phenomenon and its consequences for red giant seismology.

The next section is devoted to derivation of an asymptotic approximation for dipolar oscillations in the gravity propagation zone and a discussion of transition from standing to running waves limit. Based on that, in Sect. 3, the inner boundary condition for the acoustic oscillation of the convective envelope is formulated. Properties of the acoustic cavity in red giant envelopes are reviewed in Sect. 4. Efficiency of trapping radial and dipolar modes is discussed in Sect. 5. Numerical results on mode frequencies and damping rates caused by wave losses in selected envelope models are presented, respectively, in Sects. 6 and 7. The final section confronts theoretical results obtained in this and earlier papers with observations.

\section{An asymptotic approximation for dipolar modes}

We consider adiabatic oscillation of an isolated star ignoring all effects of rotation. The linearized momentum equation, which we write in the standard notation adopted e.g. by Aerts et al. (2010)

$\omega^{2} \rho \boldsymbol{\xi}=\boldsymbol{\nabla} p^{\prime}+\rho \boldsymbol{\nabla} \Phi^{\prime}+\rho^{\prime} g \boldsymbol{e}_{r}$,

combined with the Poisson and continuity equations leads to the familiar fourth order differential equations for the radial eigenfunctions.

That the system reduces to the second order at $\ell=0$ (radial modes) has been known from the nascent of the theory of stellar 
oscillations but that this is true also at $\ell=1$ (dipolar modes) has been demonstrated not long ago by Takata (2005). Here we we will use his formalism and mostly his notation. The four basic radial eigenfunctions $y_{k}(r)$ used in this work are defined in expressions for vectorial displacement

$\boldsymbol{\xi}=r\left[y_{1}(r) \boldsymbol{e}_{r}+y_{2}(r) \boldsymbol{\nabla}_{H}\right] Y_{\ell}^{m} \mathrm{e}^{-\mathrm{i} \omega}$

and the Eulerian perturbation of the gravitational potential

$\boldsymbol{\nabla} \Phi^{\prime}=g\left[y_{4}(r) \boldsymbol{e}_{r}+y_{3}(r) \boldsymbol{\nabla}_{H}\right] Y_{\ell}^{m} \mathrm{e}^{-\mathrm{i} \omega t}$.

Note that we have

$p^{\prime}=g \rho r\left[B y_{2}(r)-y_{3}(r)\right] Y_{\ell}^{m} \mathrm{e}^{-\mathrm{i} \omega t}$,

where

$B=\frac{\omega^{2} r}{g}$

We will use standard dimensionless coefficients calculated from stellar models

$V_{g}=\frac{g r}{c^{2}}, \quad A=-\frac{\mathrm{d} \ln \rho}{\mathrm{d} \ln r}-V_{g}, \quad$ and $\quad U=\frac{4 \pi \rho r^{3}}{M_{r}}$.

Takata showed that the radial eigenfunctions of dipolar modes satisfy the linear algebraic relation

$U B\left(y_{2}-y_{1}\right)+(2-U+B) y_{3}+(1-B) y_{4}=0$

and used it to reduce the eigenvalue problem to two first order equations for radial eigenfunctions

$z_{1}=y_{1}+\frac{y_{3}}{1-B}$

and

$z_{2}=y_{2}+\frac{y_{3}}{1-B}$.

Here, we write these equations in the following form

$r \frac{\mathrm{d}}{\mathrm{d} r}\left(\begin{array}{l}z_{1} \\ z_{2}\end{array}\right)=\left(\begin{array}{cc}V_{g}-3+S & 2-B V_{g}-S \\ 1-A / B+S & A-2-S\end{array}\right)\left(\begin{array}{l}z_{1} \\ z_{2}\end{array}\right)$,

where

$S=\frac{U B}{(1-B)^{2}}$

For the radial eigenfunctions describing perturbation we have from the definition in Eq. (3)

$r \frac{\mathrm{d} y_{3}}{\mathrm{~d} r}=(1-U) y_{3}+y_{4}$

and from Eq. (4)

$y_{4}=\frac{(U-2-B) y_{3}+U B\left(z_{1}-z_{2}\right)}{1-B}$.

At the base of red giant envelope, $B \propto r^{3} / M_{r}$ may be very small even for high frequency modes. Let us note that Eq. (7) in the $B \rightarrow 0$ limit has the same form as in the Cowling approximation but with modified definition of variables. Nearly the whole radiative core, where $A>0$, is a region of gravity wave propagation. Once the wave number, $\sqrt{2 A / B r^{2}}$, is sufficiently large an asymptotic approximation is applicable.
For any $\ell>0$ in the limit $A / B \gg 1$, the fourth order system may be asymptotically decomposed into two second order equations yielding one solution that varies rapidly with $r$ and describes a gravity wave and one that varies slowly and describes a quasi-static response to an external perturbation of the gravitational potential (Dziembowski 1971). In 1971, the goal of such a decomposition was not only to help understanding physics of nonradial oscillations in evolved stars but also to use in the interior the analytic form of the rapidly varying solution which was difficult to calculate numerically.

In that work no special attention was paid to dipolar modes for which also the slow solution may be derived in an analytical form. With Takata's equations, the solution for the potential which is divergent at $r=0$ is eliminated automatically. A superposition of the slow and rapid solutions will be used in the next section to formulate inner boundary conditions for dipolar modes trapped in the convective envelope.

The slowly varying solution of Eqs. (7) to (9), which is easily obtained by setting $z_{1}=z_{2}=0$, is

$\left(\begin{array}{l}y_{s, 1} \\ y_{s, 2} \\ y_{s, 3} \\ y_{s, 4}\end{array}\right)=\frac{C_{s}}{r}\left(\begin{array}{c}1 \\ 1 \\ -1+B \\ 2-U+B\end{array}\right)$,

where $C_{s}$ is a constant. This solution is exact and it is equivalent to one given by Takata (2006a, Eq. (116)). It describes uniform shift of star interior in response to the external potential change, which in our application is caused by oscillations in the envelope.

To obtain the rapidly varying solution, we adopt

$\left|\frac{\mathrm{d} z_{k}}{\mathrm{~d} r}\right| \sim\left|k_{r} z_{k}\right|$

where

$k_{r}=\frac{1}{r} \sqrt{\frac{2 A}{B}} \gg \frac{1}{r}$

is the radial component of gravity wave number. Setting in Eq. (7)

$z_{1}=\frac{\mathcal{Z}_{1}}{r^{3} \sqrt{\rho}}$

we obtain

$r^{2} \frac{\mathrm{d}^{2} \mathcal{Z}_{1}}{\mathrm{~d} r^{2}}+\left[\frac{2 A}{B}+O(1)\right] \mathcal{Z}_{1}=0$

The asymptotic solution of this equation is

$\mathcal{Z}_{1}=C_{r} \frac{\mathrm{e}^{\mathrm{i} \Psi}+\mathrm{e}^{-\mathrm{i} \Psi}}{\sqrt{k_{r}}}, \quad \Psi=\int_{r_{0}} k_{r} \mathrm{~d} r$,

where $C_{r}$ is another constant. If there is no convective core $r_{0}=0$ and this form is obtained by fitting to the spherical Bessel function $j_{1}(r / R)$, which is the solution of Eq. (7) valid near the center. The same form is valid for stars with convective cores but then $r_{0}=r_{\mathrm{c}}$, which is the core radius. The first and second exponential components describe, respectively, outwardly and inwardly running gravity waves. At $B \ll 1$, Eqs. (7) and (14) imply

$z_{2} \approx \frac{1}{2}\left(r \frac{\mathrm{d} z_{1}}{\mathrm{~d} r}+\left(3-V_{g}\right) z_{1}\right)=\frac{\mathcal{Z}_{2}}{2 r^{3} \sqrt{\rho}}$, 
where

$\mathcal{Z}_{2}=r \frac{\mathrm{d} \mathcal{Z}_{1}}{\mathrm{~d} r}+\frac{A-V_{g}}{2} \mathcal{Z}_{1}$

With the same accuracy, Eqs. (5) and (6) imply $y_{1}=z_{1}$ and $y_{2}=z_{2}$. Then, Eqs. (8) and (9) yield $y_{3}=-U B y_{1} / 2$ and $y_{4}=$ $(U-2) y_{3}+U B\left(z_{1}-z_{2}\right)$. Thus, for the rapidly varying solution, we get the following asymptotic approximation,

$$
\left(\begin{array}{l}
y_{r, 1} \\
y_{r, 2} \\
y_{r, 3} \\
y_{r, 4}
\end{array}\right)=\frac{C_{r}}{\sqrt{\rho} r^{3}}\left(\begin{array}{c}
2 \mathcal{Z}_{1} \\
\mathcal{Z}_{2} \\
-U B \mathcal{Z}_{1} \\
-U B\left[\mathcal{Z}_{2}+(U-4) \mathcal{Z}_{1}\right]
\end{array}\right) .
$$

If energy losses in the interior are negligible, the amplitudes of the inwardly and outwardly running waves are equal and the solution is a standing wave. In luminous red giants even at frequencies as high as the acoustic cut-off the phase at the top gravity propagation zone is large. Thus, all nonradial modes have mixed character. The distance between consecutive modes of the same $\ell \geq 1$ is much less than the distance between consecutive radial modes (the large frequency separation) and decreases during evolution along the RGB. The relative amplitude in the interior is modulated in the rhythm determined by the acoustic frequencies of the envelope. With increasing luminosity, the amplitude of modulation increases which leads to the appearance of modes trapped in the envelope. This happens first for modes of higher degrees but ultimately also for $\ell=1$. Such modes are of our interest.

The most important evolutionary effect on nonadiabatic properties of nonradial oscillations is the onset of significant damping in the inner part of the radiative interior (Dziembowski 1971). In the first works on excitation of nonradial modes in giants (Dziembowski 1977; Osaki 1977), only unfitted envelope models were considered. Solutions of the nonadiabatic problem were obtained upon neglecting perturbation of the gravitational potential (the Cowling approximation). One needed boundary condition was obtained by neglecting the reflected wave. The justification was that radiative damping in the deep interior causes that the reflected wave returns with much reduced amplitude and may be ignored in some cases, such as low order modes of moderate degrees in Cepheids and RR Lyrae stars considered in those papers. The modes were found unstable in spite of large damping in the interior because the amplitudes there were very low as a result of efficient trapping in the envelope. For luminous red giants, such a situation was found also in the case of dipolar modes (Dziembowski et al. 2001).

Complex eigenfrequencies determined with the running wave boundary condition set in the envelope yield approximate values for true stellar eigenfrequencies if $\left|\Psi_{I}\left(r_{b}\right)\right| \gg 1$, where subscript $I$ denotes the imaginary part of a complex quantity (we will use subscript $R$ to denote the real part) not only for unstable modes but also for stochastically driven stable modes. The requirement is that the damping rate is not too high. The explicit condition is easy to write in the case when the quasi-adiabatic approximation is still applicable and $\left|\omega_{I}\right| \ll \omega_{R}$. In such a special but not unrealistic case we have (e.g. Dziembowski et al. 2001)

$k_{r} \simeq \frac{\sqrt{\ell(\ell+1)}}{\omega_{R}} \frac{N}{r}\left[1-\mathrm{i}\left(D+\frac{\omega_{I}}{\omega_{R}}\right)\right]$,

where

$D=\frac{\ell(\ell+1)}{8 \pi \omega^{3}} \frac{g L_{\mathrm{r}}}{r^{4} p} \frac{\nabla_{\mathrm{ad}}}{\nabla}\left(\nabla_{\mathrm{ad}}-\nabla\right)$, and this condition is

$-\Psi_{I}\left(r_{b}\right)=\int_{0}^{\Psi_{b}}\left(D-\frac{\omega_{I}}{\omega_{R}}\right) \mathrm{d} \Psi_{R} \gg 1$,

where $\Psi_{b}=\Psi_{R}\left(r_{b}\right)$. The trapping effect follows essentially from adiabatic properties of stellar oscillation but it is enhanced by nonadiabatic effects in the case of unstable or weakly stable modes.

As long as we stay within the domain of linear oscillation theory, the use of running wave solution in the situation when the strong inequality in Eq. (18) is not satisfied means an overestimate of damping in the core. However, it must be kept in mind that nonlinear effects may significantly enhance radiative damping. Could such effects be of any importance in the case of stochastically driven oscillations? Dupret et al. (2009) found that everywhere in their models $|(\xi \cdot \nabla) \xi| \ll|\xi|$ and gave the negative answer. However, their argument refers to a strong nonlinearity, whereas at much lower amplitudes the resonant excitation of high degree modes may occur which leads to a significant increase of the damping rates (e.g. Kumar \& Goodman 1996). Unfortunately, in this case, a precise determination of the condition for the running wave approximation is not easy.

In this paper, we adopt the running wave boundary condition at the top of radiative interior. This means that we treat the convective envelope as a separate oscillating body loosing part of its energy by emitting gravity wave from its bottom. We will examine implication of this assumption and appeal to observations to verify its applicability.

\section{The boundary conditions and eigenfrequencies}

The asymptotic approximation developed in the previous section allows to formulate the inner boundary condition which may be imposed on numerical solutions of the equations for dipolar oscillation in the outer layers. Radiative dissipation arises mainly deep in the star interior, so that even when it is large, we may still rely on adiabatic approximation in the outer part of the g-mode propagation zone, where we want to apply the inner boundary condition. This is not an essential approximation and in fact it was not used in Dziembowski (1977) paper. However, we will avoid the Cowling approximation, which may be globally justified at higher degrees but not, as Christensen-Dalsgaard $\&$ Gough (2001) stressed, at $\ell=1$. We are now going to correct this mistake by taking into account perturbation of the gravitational potential and the slowly varying solution.

The general solution of Eqs. (7) to (9) is a linear superposition of the slow and rapid solutions given in Eqs. (10) and (17), respectively. It involves two unknown constants and thus yields two independent boundary conditions. The slow solution has the form specific for dipolar modes and only for these modes it is independent of the interior structure. The form of the rapid solution is valid for any $\ell$ if in Eq. (11) for $k_{r}$ the number 2 is replaced with $\ell(\ell+1)$. For the running wave solution, we get from Eqs. (14) and (16)

$\mathcal{Z}_{2}=\left[\mathrm{i} r k_{r}+\frac{1}{2}\left(A-V_{g}+\frac{r}{\left|k_{r}\right|} \frac{\mathrm{d}\left|k_{r}\right|}{\mathrm{d} r}\right)\right] \mathcal{Z}_{1}$.

The boundary condition obtained in this way may be applied at any place within the radiative interior, where the asymptotic approximation is valid. The explicit form of the inner boundary condition for the case of running wave is given later in this section. The same conditions may be applied at the base of convective envelope, $r=r_{b}$, if $B\left(r_{b}\right) \ll 1$ and there is a jump in $A$ to 
a finite value due to discontinuity in hydrogen abundance. Note that then a corresponding discontinuity of $y_{2}$ must be taken into account. However, it is more realistic to treat $A(r)$ as a steep but continuous function. In such a case, some modifications in the form of boundary are needed.

To this end, we consider thin layer beneath the envelope bottom where $A$ rises from zero at $r=r_{b}$ to a finite value at its lower boundary where $A / B$ becomes large. Like before, we start with Eq. (13) but now we seek its asymptotic solution in the $B \rightarrow 0$ limit in terms of the Airy function $\mathcal{A} i$ and $\mathcal{B} i$ (see e.g. Eq. (10.4.112) in Abramovitz \& Stegun 1972). The solution is

$\mathcal{Z}_{1}=C_{A} \mathcal{A} i(s)+C_{B} \mathcal{B} i(s)$.

where

$s=-\lambda^{2 / 3}\left(r_{b}-r\right), \quad \lambda^{2}=-\left(\frac{2}{r^{2} B} \frac{\mathrm{d} A}{\mathrm{~d} r}\right)_{b}>0$.

Note that

$r \frac{\mathrm{d} \mathcal{Z}_{1}}{\mathrm{~d} r}=\beta \frac{\mathrm{d} \mathcal{Z}_{1}}{\mathrm{~d} s}$

where

$\beta \equiv r \lambda^{2 / 3}=\left(-\frac{2 r}{B} \frac{\mathrm{d} A}{\mathrm{~d} r}\right)_{b}^{1 / 3}$.

At $\left(r_{b}-r\right) \kappa \gg r_{b}$,

$\mathcal{Z}_{1} \propto|s|^{-1 / 4}\left(C_{A} \sin \Psi+C_{B} \cos \Psi\right), \quad \Psi=\frac{2}{3}(-s)^{\frac{3}{2}}+\frac{\pi}{4}$.

Thus, for the inwardly running wave we must have $C_{A}=-\mathrm{i} C_{r}$, $C_{r} \equiv C_{B}$, and we get

$\mathcal{Z}_{1}=\mathcal{B i}(s)-\mathrm{i} \mathcal{A} i(s)$.

The expression for $z_{2}$ in Eq. (15) remains valid but with

$\mathcal{Z}_{2}=\beta \frac{\mathrm{d}}{\mathrm{d} s}(\mathcal{B} i-\mathrm{i} \mathcal{A} i)+\frac{A-V_{g}}{2} \mathcal{Z}_{1}$

Again with Eqs. (8) and (9), we may derive the corresponding expressions for $y_{3}$ and $y_{4}$. The slow solution given by Eq. (10) remains unchanged. For the explicit form of the solution at convective envelope bottom, we use the values of the Airy functions and their derivatives at $s=0$,

$\mathcal{A} i=\frac{\mathcal{B} i}{\sqrt{3}}, \quad \frac{\mathrm{d} \mathcal{A} i}{\mathrm{~d} s}=\frac{-1}{\sqrt{3}} \frac{\mathrm{d} \mathcal{B} i}{\mathrm{~d} s}, \quad \frac{1}{\mathcal{B} i} \frac{\mathrm{d} \mathcal{B} i}{\mathrm{~d} s}=3^{1 / 3} \frac{\Gamma(2 / 3)}{\Gamma(1 / 3)}=0.729$,

and $A\left(r_{b}\right)=0$.

At $r=r_{b}$, with the use of Eqs. (10), (17), (22), and (23), we finally obtain

$\left(\begin{array}{l}y_{1} \\ y_{2} \\ y_{3} \\ y_{4}\end{array}\right)=\tilde{C}_{s}\left(\begin{array}{c}1 \\ 1 \\ -1+B \\ 2-U+B\end{array}\right)+\tilde{C}_{r}\left(\begin{array}{c}2 \mathcal{Z}_{1} \\ \mathcal{Z}_{2} \\ -U B \mathcal{Z}_{1} \\ -U B\left[\mathcal{Z}_{2}+(U-4) \mathcal{Z}_{1}\right]\end{array}\right)$

where

$\tilde{C}_{s}=\frac{C_{s}}{r_{b}}, \quad \tilde{C}_{r}=\frac{C_{r}}{\left(r^{3} \sqrt{\rho}\right)_{b}}$,

$\mathcal{Z}_{1}=1-\frac{\mathrm{i}}{\sqrt{3}}, \quad$ and $\quad \mathcal{Z}_{2}=0.729 \beta\left(1+\frac{\mathrm{i}}{\sqrt{3}}\right)-\frac{V_{g}}{2} \mathcal{Z}_{1}$.

A83, page 4 of 10
The same form is valid for the inner boundary condition applied within the radiative core but, as follows from Eq. (19), with $\mathcal{Z}_{1}=1$, and

$\mathcal{Z}_{2}=\left(\mathrm{i} k_{r}+\frac{A_{-} V_{g}}{2}\right) \mathcal{Z}_{1}$

Two inner boundary conditions in the form of linear algebraic relations are obtained by eliminating constants $\tilde{C}_{s}$ and $\tilde{C}_{r}$. The relations are complex and so are the resulting eigenvalues and eigenfunctions even if, as we will do in this paper, all nonadiabatic effects in the envelope are ignored.

Derivation of the outer boundary conditions for oscillations in realistic stellar models cannot be done in such a rigorous way as in the case of polytropic models considered by Takata (2005). In realistic models, $V_{g}(R)<\infty$, so there is no singularity, and $U>0$. In main sequence stars similar to the Sun, $V_{g} \sim A \sim 10^{3}$ and $U \sim 10^{-6}$. In stars near the tip of the RGB, $V_{g} \sim A \sim 10^{2}$ and $U \sim 10^{-3}$. The standard procedure is to set the boundary condition in the atmosphere near maximum of $V_{g}$ and ignore $U$. Then in an equation like Eq. (7), the coefficients are approximately constant and for the wave reflected downward we have

$B y_{2}=\left[1-\frac{\Gamma_{1}}{2}\left(1-\sqrt{1-\left(\frac{\omega}{\omega_{\mathrm{ac}}}\right)^{2}}\right)\right] y_{1}$,

where $\omega_{\mathrm{ac}} \equiv g \Gamma_{1} / 2 c$ is the acoustic frequency. The vacuum solution of the Poisson equation outside the star implies $y_{4}=-2 y_{3}$ at $r=R$. This is the second outer boundary condition adopted in this paper. For dipolar modes, an additional condition follows from Eq. (4), which combined with the previous two yields $y_{3}=U\left(y_{1}-y_{2}\right) / 3$. Thus, we have three conditions and, with the standard normalization $y_{1}=1$, we may carry a single integration from the surface downward. Application of this standard procedure to luminous red giants may seem problematical and therefore the sensitivity of results to variation in the outer boundary condition must be checked. Only one of the inner boundary conditions is needed in a search for eigenfrequency. The second condition may be used as a test of numerical accuracy.

\section{The acoustic propagation zone}

It is well-known that frequencies of low order radial modes in evolved stars may be accurately calculated from their envelope models alone. We will see that in the case of luminous red giants, this property extends up to the highest orders. The bottom of the envelope, which must cover the whole acoustic propagation zone, may still be within the convective region. Thus, what determines the depth of the propagation zone is not the $\mathcal{N}(r)=\omega$ condition, where $\mathcal{N}$ is the Brunt-Väisälä frequency. A general condition limiting the propagation zone from its bottom does not exist ${ }^{1}$. The condition $\omega<\omega_{\text {ac }}$ for wave reflection is derived for an isothermal plane-parallel atmosphere built of perfect gas. Although in this case the condition is numerically close to $\omega<\mathcal{N}$, its origin is different (to see this consider, e.g., the case of gas with $\Gamma_{1}=1$ when $\mathcal{N}=0$ ). In fact, the condition follows from general rule prohibiting wave propagation if the background structure changes significantly over wavelength. The equality $\omega=\omega_{\mathrm{ac}}$ corresponds to equality of the radial wave

1 The generalized acoustic cut-off frequency, $\omega_{c}$, as defined e.g. in (Aerts et al. 2010) is not a good choice in the present application because $\omega_{\mathrm{c}}^{2}<0$ in the lower part of the envelope. 
Table 1. Selected models from the BaSTI track at $M_{0}=2 M_{\odot}$ and $Z=0.02$.

\begin{tabular}{ccccccc}
\hline \hline & $\begin{array}{c}M \\
\left(M_{\odot}\right)\end{array}$ & $\begin{array}{c}\log L \\
\left(L_{\odot}\right)\end{array}$ & $\begin{array}{c}\log T_{\text {eff }} \\
(\mathrm{K})\end{array}$ & $r_{b} / R$ & $\begin{array}{c}v_{\max } \\
(\mu \mathrm{Hz})\end{array}$ & $\begin{array}{c}\Delta v \\
(\mu \mathrm{Hz})\end{array}$ \\
\hline 1 & 1.998 & 1.500 & 3.692 & 0.0746 & 110.8 & 8.851 \\
2 & 1.997 & 2.000 & 3.663 & 0.0454 & 27.47 & 3.071 \\
3 & 1.994 & 2.501 & 3.628 & 0.0287 & 6.51 & 1.050 \\
4 & 1.985 & 3.001 & 3.586 & 0.0104 & 1.46 & 0.329 \\
5 & 1.967 & 3.355 & 3.552 & 0.0061 & 0.49 & 0.137 \\
6 & 1.967 & 1.771 & 3.687 & 0.2410 & 55.90 & 5.752 \\
\hline
\end{tabular}

number acoustic waves to $0.5 / H_{p}$, where $H_{p}=p / g \rho$ is the pressure distance scale. It does not seem unreasonable to use $\omega<\omega_{\text {ac }}(r)$ as a crude local no-propagation condition, as long as $H_{p}$ is the shortest of all local distance scales.

For nonradial modes the acoustic propagation zone may be limited from the bottom by the Lamb frequency, $\mathcal{L}_{\ell}=$ $\sqrt{\ell(\ell+1)} / r$ exceeding $\omega$. This critical frequency was determined upon assuming the Cowling approximation. Avoiding this approximation, Takata (2006b) found a simple modification of the Lamb frequency for dipolar modes, which in our notation may be written as

$\mathcal{L}_{1 m}=\mathcal{L}_{1}\left(1-\frac{U}{3}\right)$

In our application, this modification is not very significant. Near the bottom of convective envelope the correction is at most one percent. The local maximum of $U / 3$ (up to 0.5 ) is reached in the middle of the envelope. We will not use modified $\mathcal{N}$, where the same factor appears in the denominator, because it has no effect on lower boundary of the acoustic propagation zone.

During evolution along the RGB frequencies of stochastically excited oscillations decrease. The critical frequencies $\omega_{\mathrm{ac}}(r)$ and $\mathcal{L}_{1 m}(r)$ at specified fractional radius decrease too, but not as fast. Therefore the bottom of the acoustic propagation zone moves upward. In the same time, the bottom of the convective envelope moves downward. This leads to expansion of the evanescent zone between the gravity and acoustic propagation zones and to efficient mode trapping in the convective envelope.

As an example. we consider a sequence of red giant envelope models with mass, $M$, luminosity, $L$, and effective temperature, $T_{\text {eff }}$, taken from BaTSI (Pietrinferni et al. 2006) evolutionary tracks for the initial mass $M_{0}=2 M_{\odot}$ and heavy element abundance parameter $Z_{0}=0.02$. The opacity and equation of state data come from the same source. The adopted mixing length parameter $\alpha=1.85$ corresponds to 1.74 in BaTSI models. Parameters of these models are listed in Table 1. The models may be regarded realistic down to the bottom of the convective envelope. Listed in the table values of $v_{\max }$, corresponding to the expected maximum of acoustic power for stochastic oscillations, were evaluated with the Kjeldsen \& Bedding (1995) expression

$v_{\max }=\frac{M}{L}\left(\frac{T}{T_{\odot}}\right)^{3.5} \times 3050 \mu \mathrm{Hz}$.

The values of $\Delta v$, which denote the distances between consecutive radial modes (the large separations), were calculated around $v_{\max }$ for reconstructed envelope models with Warsaw codes (see e.g. Dziembowski \& Soszyński 2010). Models 1 to 5 represent stars ascending the RGB. Model 6 in Table 1 represents a helium burning (red clump) object
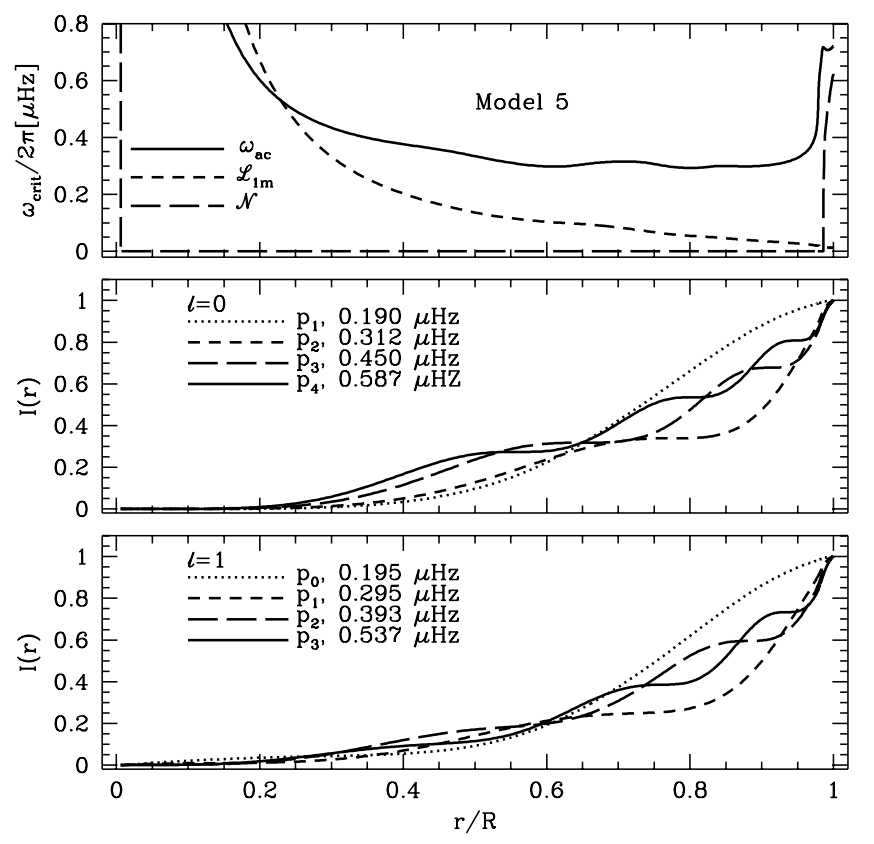

Fig. 1. Critical frequencies (see text) in Model 5 (see Table 1). (Top) Relative contributions to mode inertia integrated upward from the base convective envelope for all four modes with frequencies below $\omega_{\mathrm{ac}}(R)$. (Middle) The same for the first four dipolar modes trapped in the envelope. (Bottom) There is one more dipolar overtone, whose frequency $\nu=0.677 \mu \mathrm{Hz}$ is below $\omega_{\mathrm{ac}}(R)$.

We begin with the extreme case, that is, with Model 5, which is located at the tip of the RGB. The three critical frequencies in this model are shown in the top panel of Fig. 1. The acoustic cavity for radial mode is determined solely by the run of $\omega_{\mathrm{ac}}$. For dipolar modes the bottom of the acoustic propagation zone is determined by

$\omega=\max \left[\omega_{\mathrm{ac}}(r), \mathcal{L}_{1 m}(r)\right]$

condition and the top of the gravity propagation zone by $\omega=\mathcal{N}$. In the middle and bottom panels, we show fractional inertia

$I(r)=\int_{r_{b}}^{r}\left(\left|y_{1}\right|^{2}+2\left|y_{2}\right|^{2}\right) \rho r^{4} \mathrm{~d} r$

for radial and dipolar modes, respectively. For these plots, normalization $I \equiv I(R)=1$ was adopted. With the standard normalization $y_{1}(R)=1$, the value of $I \equiv I(R)$ is a measure of mode trapping. Let us stress that $I$ is not the total mode inertia but only its part contributed by the convective envelope. The plots may suggest that for any of the modes shown in Fig. 1, there is no contribution to the total inertia (energy) from the interior below $r \approx 0.2$. In fact, for the dipolar modes the inertia, $I_{\mathrm{c}}$, arising from the slow component, which keeps the center of mass of the whole star at rest, is substantial. This contribution to the total inertia may be calculated without any knowledge of the core structure. Using in Eq. (27) the expressions for $y_{1}$ and $y_{2}$ from Eq. (10), we get

$I_{\mathrm{c}} \equiv \int_{0}^{r_{b}}\left(\left|y_{s, 1}\right|^{2}+2\left|y_{s, 2}\right|^{2} \rho r^{4} \mathrm{~d} r=3\left(\frac{\rho r^{5}}{U}\right)_{b}\left|\tilde{C}_{s}\right|^{2}\right.$.

The $I_{\mathrm{c}} / I$ ratio ranges up to $1 / 3$ for the $p_{0,1}$ mode and decreases with the mode order.

According to the terminology adopted in works on stellar pulsation, the radial $p_{1}$ is called fundamental (no nodes between 

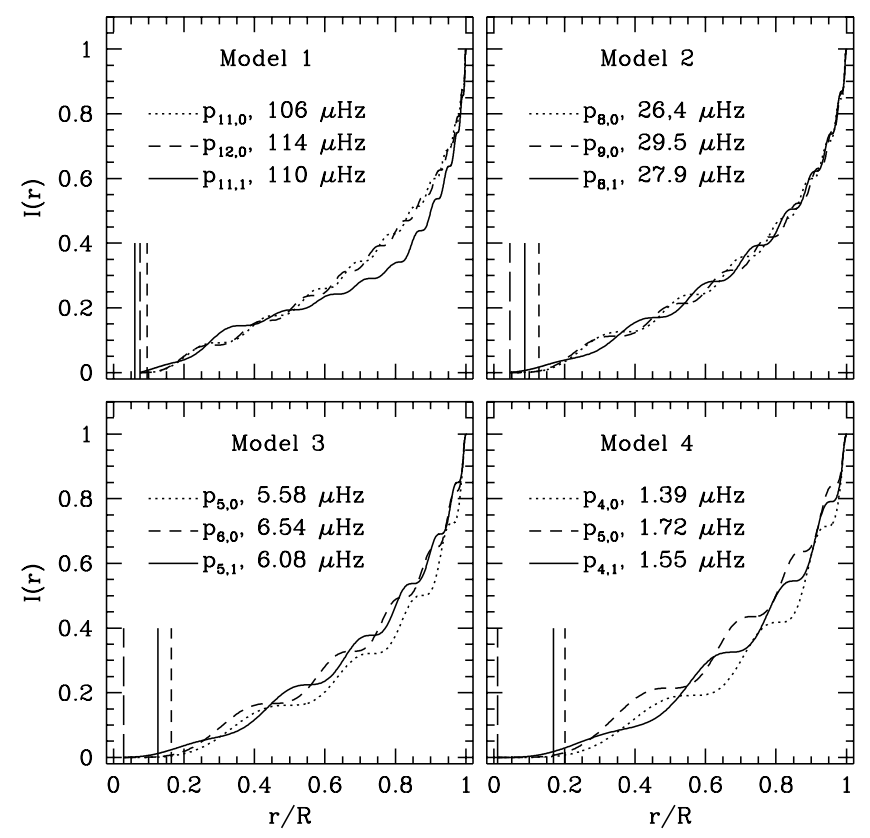

Fig. 2. Relative contributions to mode inertia integrated upward from the base convective envelope for three modes (two radial and one dipolar) with frequencies close to $v_{\max }$ in the four indicated models whose parameters are given in Table 1. The short lines parallel to the ordinate axis mark the bottom of the convective envelope (long-dashed style), the places where $2 \pi v_{\max }=\omega_{\mathrm{ac}}$, and $\mathcal{L}_{1 m}$ (drawn in the solid, and shortdashed style, respectively). The styles correspond to those used to show the respective frequencies in Fig. 1.

the surface and the lower boundary and one zone of steep rise in $I)$. Its frequency is well below the minimum value of $\omega_{\mathrm{ac}}(r)$. For the overtones, most of the contribution to $I$ arises in the zone $\omega \lesssim \omega_{\mathrm{ac}}(r)$ condition or close to it. Comparison of middle and bottom panels reveals first of all a similarity of the energy distribution in dipolar and radial modes, particularly in case of first (fundamental) modes whose frequencies are close. More differences are seen for the overtones.

Let us note in the top panel that the critical frequencies $\omega_{\mathrm{ac}}(r)$ and $\mathcal{L}_{1 m}(r)$ are close to each other in the frequency range of the dipolar overtones depicted in the panel. The role of the Lamb frequency is reflected in the shape of $I(r)$ for all dipolar overtones. Beginning with $\mathrm{p}_{3}$, this critical frequency determines the bottom of the acoustic propagation zone.

The place between consecutive radial modes at which the dipolar mode appears depends on the critical frequencies determining the lower boundary of the acoustic propagation zone. If it is $\omega_{\mathrm{ac}}$, which is $\ell$-independent, the dipolar and radial modes have close frequencies. This is the case for the low order modes in Model 5, as we may see in numbers quoted in Fig. 1. With increasing oscillation frequency, the role of the Lamb frequency increases and the dipolar mode moves away but is still quite far from the mid point between consecutive radial modes. It comes closer to this point in less luminous stars where higher order modes occur near $v_{\max }$.

Figure 2 shows in separate panels fractional moment of inertia for modes with frequencies around $v_{\max }$ in Models 1 to 4. The places where the critical frequencies are equal to $v_{\max }$ are marked in each panel. The bottom of the acoustic cavity is clearly within the convective envelope except for Model 1, where the equality $\omega_{\mathrm{ac}}=2 \pi v_{\max }$ takes place in the radiative core. For the second critical frequency, the equality occurs in the envelope but the evanescent zone is too narrow for an efficient mode

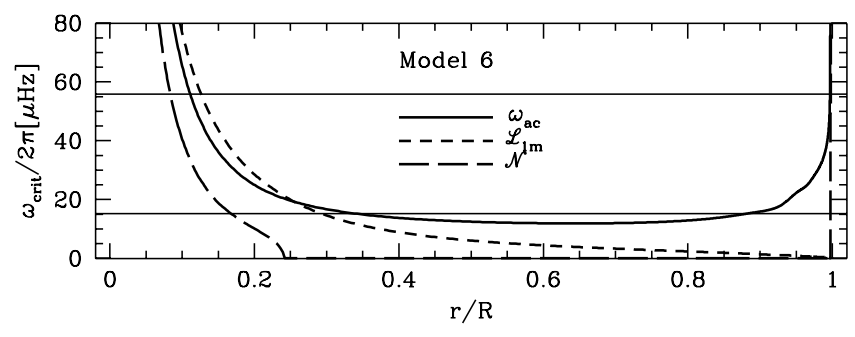

Fig. 3. Critical frequencies in Model 6. The two horizontal lines correspond to dipolar mode frequencies. The lower one to $p_{1,1}$ and the upper to $p_{9,1}$, whose frequency is near $v_{\max }$.

trapping of the dipolar mode within the convective envelope. We may observe in this figure how the width of the evanescent zone increases with stellar luminosity leading to trapping $\ell=0$ and 1 modes in the upper part of the envelope. This is seen in the upward shifts of the oscillatory pattern in $I(r)$.

Figure 3 shows critical frequencies in Model 6 representing an object in the phase of He burning in the convective core. The convective envelope is relatively shallow and the $\mathcal{L}_{1 m}=2 \pi v_{\text {max }}$ equality takes place in the radiative part of the interior. In Sect. 3, we described the version of the running wave boundary condition, which may be applied in the gravity wave propagation zone. However, it is rather unlikely that the assumption that the reflected wave has negligible amplitude might be justified in this case.

\section{Mode trapping}

Mode trapping in the acoustic cavity is a result of wave reflection within the evanescent zone. The efficiency of this process depends on the width of this zone and on mode degree. The eigenfunction in the evanescent zone is a superposition of monotonically increasing and decreasing components. At higher degrees, the dependence of the displacement on the distance to the center in the two components is approximately given by $r^{\ell-1}$ and $r^{-(\ell+2)}$. The large contrast between these behaviors causes one of the component to dominate and it is the increasing component for modes trapped in the envelope. A narrow evanescent zone suffices for an efficient trapping. We may speak about hard reflection. This effect is behind the occurrence of unstable modes of moderate $\ell$ in models of Cepheids and RR Lyrae stars. At $\ell=0$ and 1 there are also monotonically increasing and decreasing components but not as fast as at higher degrees. The reflection is softer and a wider evanescent zone is needed for an efficient trapping.

For radial modes, efficient trapping manifests itself in a weak dependence of frequencies on the exact form of the inner boundary condition set at the bottom of the convective envelope. This is so because then the contribution from the decreasing component becomes very small once the acoustic cavity is reached and thus has a negligible influence on eigenfrequencies. The effect may be quantified by considering the generic form of the boundary condition for homogenous second order equations

$\cos (\pi \phi) r \frac{\mathrm{d} y_{1}}{\mathrm{~d} r}+\sin (\pi \phi) y_{1}=0$

and letting $\phi$ vary between 0 and 1 . The numbers quoted in Figs. 1 and 2 were obtained assuming $\phi=0$. In Fig. 4 the effect is illustrated for Models 1 and 2. In the former model, except of a narrow range, the value of $\phi$ has almost no effect on frequencies. In this range, the boundary condition allows a large 
W. A. Dziembowski: Dipolar modes in luminous red giants
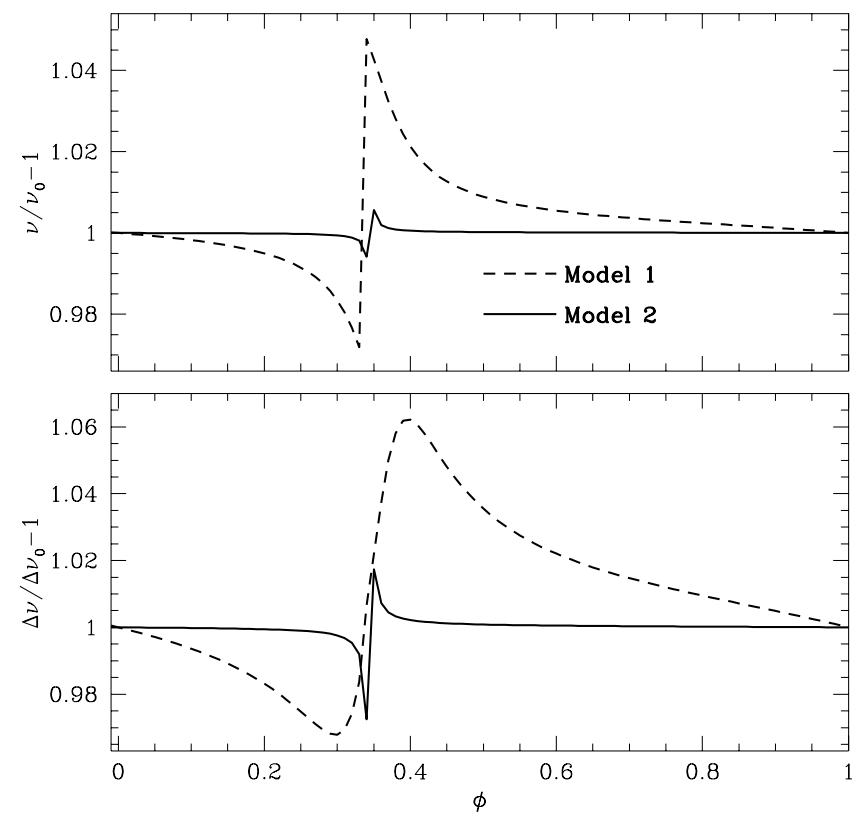

Fig. 4. Dependence of radial mode frequencies, $v$, on the parameter $\phi$ adopted in Eq. (29). The pairs of frequencies around $v_{\max }$ in Models 1 and 2 are considered. Plotted are the shifts relative to the values at $\phi=0$. The top panel shows the shift in the lower frequency. The bottom panel shows the shift in the frequency difference.

decreasing component which is certainly impossible for any realistic model of the whole star. With the $y_{1}\left(r_{b}\right)=0$ condition we are safe away from this bad range. In Model 1 the effect of varying $\phi$ is considerably larger which is consistent with what we see in Fig. 2. In this model, the propagation zone for the two radial modes extends to the core.

Changes in dipolar mode trapping may be observed by relaxing the inner boundary condition and treating $I$, defined in Eq. (27), as a continuous function of frequency. The top panel of Fig. 5 shows the values of $I$ in the frequency range centered at $v_{\max }$ for Models 1 and 2. The inertias of radial modes in the same frequency range are shown for a comparison. It is important to notice the difference between the two models. The minima for Model 2 are far deeper than in Model 1. The difference in trapping is better visible in the lower panel showing values of $\left|y_{1}+y_{3}\right|$ at the lower boundary. In this combination of the eigenfunctions, the contribution of the slow component in $\xi_{r}$ is nearly eliminated, as we may see in Eq. (24). In this case the minimum value is zero. The dependence is so steep in the case of luminous red giants that unfitted envelope models suffice to determine frequencies of expected dipolar mode frequencies at specified stellar parameters.

The transition to efficient trapping occurs at $\log L \approx 2$. Luminosity of our Model 1 places it between Models A and B of Dupret et al. (2009) while that of Model 2 between their Models $\mathrm{B}$ and $\mathrm{C}$. In the latter model $(\log L=2.1)$ they find that damping in radiative interior is high enough to reduce amplitudes of all dipolar modes below the detection level, except the ones corresponding to $I$ minima. The value of the critical luminosity refers to models with $M_{0}=2$ and it is lower at lower mass.

\section{Frequencies of the trapped modes}

The minima of $\left|y_{1}+y_{3}\right|_{b}$ shown in Fig. 5 occur at frequencies close to real part of the eigenfrequencies obtained with the code

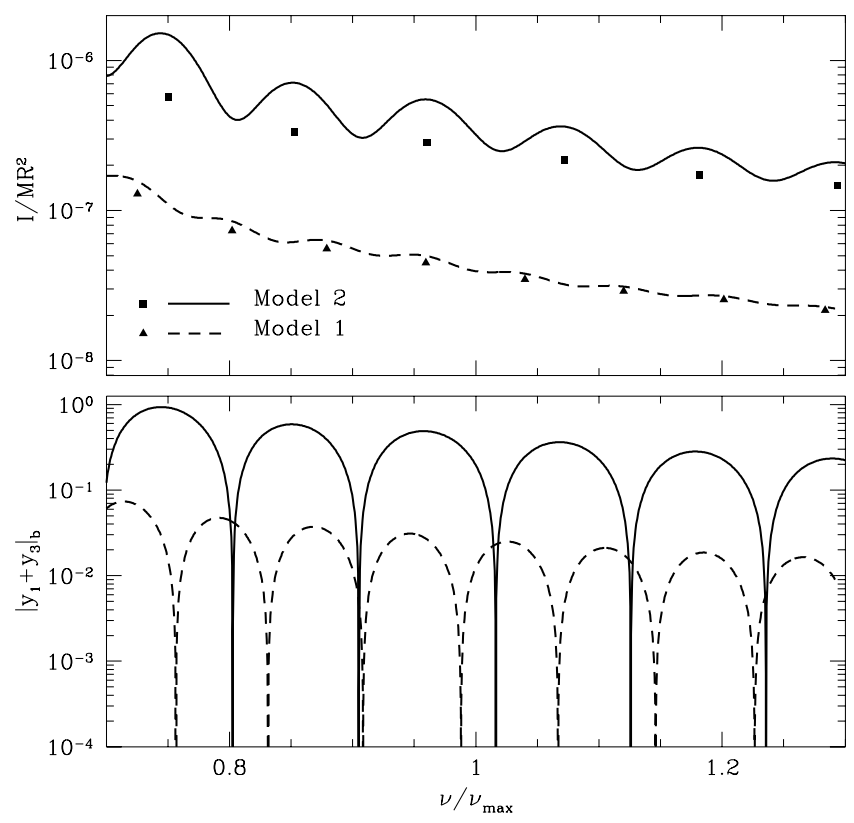

Fig. 5. Inertias of dipolar and radial modes in the frequency range centered at $v_{\max }$ in Models 1 and 2 are shown in the top panel. For dipolar modes the inner boundary condition was relaxed so that inertia is a continuous function of frequency. The standard outer boundary condition and normalization were adopted in all the cases. The quantity plotted in the bottom panel is a measure of the gravity wave amplitude at the top of the propagation zone.

described in Sect. 3. The location of the minima and the dipolar mode frequencies close to the mid points between consecutive radial mode frequencies is expected in the $B \rightarrow \infty$ limit. Note that in this limit $S \rightarrow 0$ and Eq. (7) takes again the form corresponding to the Cowling approximation, which is the basis of the usual asymptotic expressions for dipolar mode frequencies. However, asymptotic expressions yield a good approximation for eigenfrequencies only if at the place where $\mathcal{L}_{1}(r)=2 \pi v$, we have $|S| \ll 1$ and this is not true in most the cases considered in this paper. Beginning with $\ell=2$, the Cowling approximation is applicable and the asymptotic expressions may be derived in the usual way.

The real parts of eigenvalues obtained in the way described in Sect. 3 differ very little from eigenfrequencies obtained with the simple real inner boundary condition $y_{1}\left(r_{b}\right)=0$. Only in Model 1 the difference exceeds one percent.

The small separations based on dipolar and radial mode frequencies are one of standard characteristics of stochastic oscillations. Here, we use normalized small separations defined as

$\delta_{n} \equiv \frac{\delta v_{0,1}}{\Delta v}=\frac{0.5\left(v_{n+1,0}+v_{n, 0}\right)-v_{n, 1}}{v_{n+1,0}-v_{n, 0}}$,

which measure the departure of dipolar mode frequencies from the mid position between consecutive radial modes. In the top panels of Figs. 6 and 7 we show values of $\delta_{n}$ for modes at $v \approx v_{\max }$ and $n=1$, respectively. The values were calculated for envelope models with the surface parameters taken from BaSTI tracks for metallicity parameter $Z=0.02$ and initial masses $M_{0}=1$ and $2 M_{\odot}$. In Table 2 we list selected parameters for selected models at $M_{0}=1 M_{\odot}$. Similar data for models at $M_{0}=2 M_{\odot}$ and nearly the same $L$ were given earlier in Table 1 .

The jump-like changes, visible in the first figure, result from gradual switches of mode orders, from $n=8$ to 3 and from $n=12$ to 4 for $M=1$ and $2 M_{\odot}$, respectively. The changes 


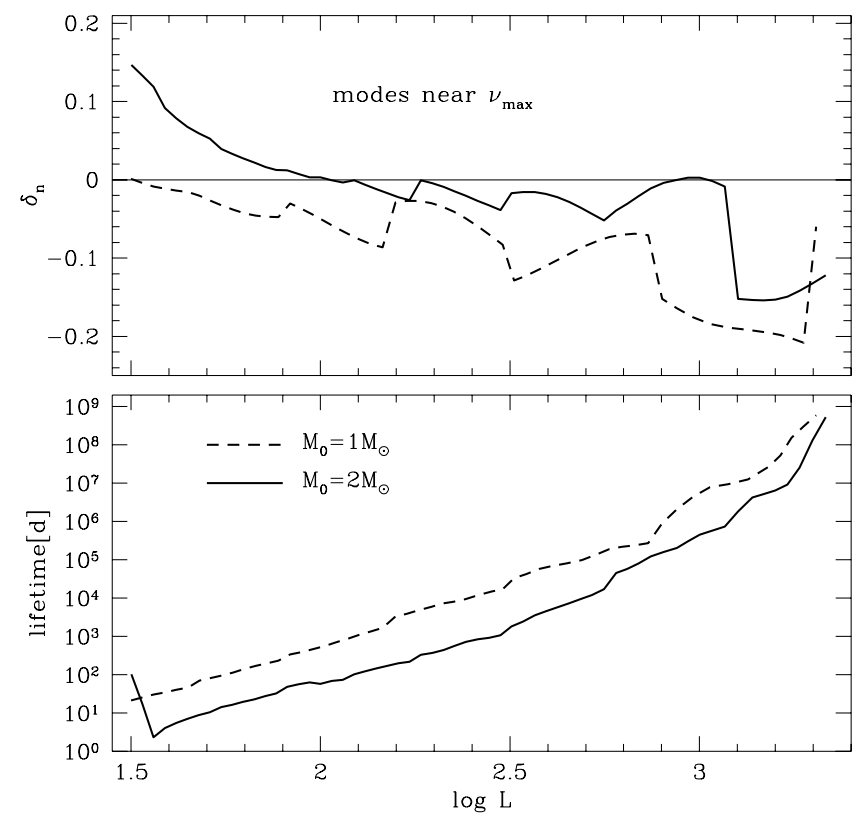

Fig. 6. The normalized small separation defined in Eq. (30) (top) and the lifetimes limited only by wave energy loss (bottom) for the dipolar mode with frequencies closest to $v_{\max }$ in red giant envelope of indicated luminosity and initial mass.

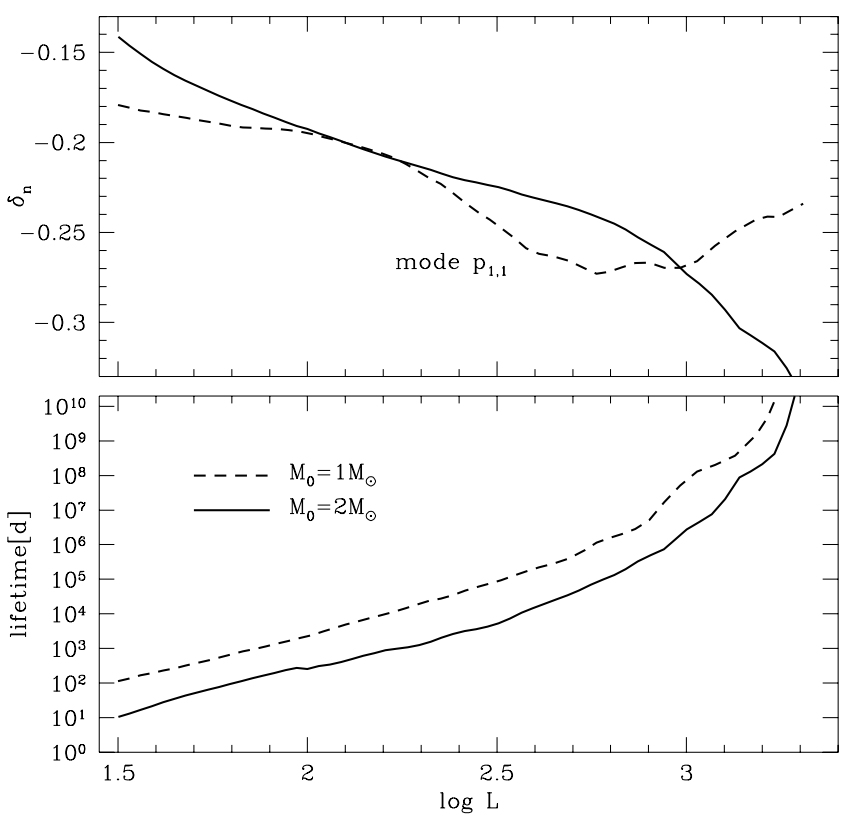

Fig. 7. The same as in Fig. 6 but for $p_{1,1}$ mode.

at constant $n$, as we may see in Fig. 6, are smooth but not necessarily monotonic. At $n=1$, the dipolar mode occurs always significantly closer to the higher frequency radial mode. Clearly, negative values of $\delta_{n}$ prevail also for modes near $v_{\max }$. Only in the low luminosity models at $M_{0}=2 M_{\odot}$ the sign is opposite. This agrees with results of Montalban et al. (2010). Negative values of $\delta_{n}$ were also found in the fit to the CoRoT data (Mosser et al. 2010). Significant departure from the mid position means that $\delta_{n}$ is an independent seismic observable, which may perhaps be measured with future, more precise data. As for today, only mixed dipolar modes give us new seismic information about
Table 2. Selected models from the BaSTI track at $M_{0}=1 M_{\odot}$ and $Z=0.02$.

\begin{tabular}{ccccccc}
\hline \hline & $\begin{array}{c}M \\
\left(M_{\odot}\right)\end{array}$ & $\begin{array}{c}\log L \\
\left(L_{\odot}\right)\end{array}$ & $\begin{array}{c}\log T_{\mathrm{eff}} \\
(\mathrm{K})\end{array}$ & $r_{b} / R$ & $\begin{array}{c}v_{\max } \\
(\mu \mathrm{Hz})\end{array}$ & $\begin{array}{c}\Delta v \\
(\mu \mathrm{Hz})\end{array}$ \\
\hline 1 & 0.997 & 1.500 & 3.658 & 0.0628 & 41.53 & 4.869 \\
2 & 0.994 & 2.000 & 3.628 & 0.0410 & 10.31 & 1.717 \\
3 & 0.986 & 2.501 & 3.590 & 0.0213 & 2.38 & 0.586 \\
4 & 0.964 & 3.005 & 3.542 & 0.0125 & 0.49 & 0.172 \\
5 & 0.933 & 3.275 & 3.511 & 0.0132 & 0.20 & 0.092 \\
\hline
\end{tabular}

red giants beyond $\Delta v$ and $v_{\max }$. We will return to this matter in Sect. 8 .

\section{Effect of wave losses on mode lifetime}

The imaginary part of the eigenfrequency calculated with our code yields the damping rate, $\gamma=-\omega_{I}$, which takes into account only the effect of the wave losses at the bottom of the convective envelope. Of course, this is not the only important source of damping but the other resides predominantly in the outermost layers, where $\omega_{\mathrm{ac}} \gg \mathcal{L}_{\ell}$ and the eigenfunctions depend mainly on mode frequency and not on its degree. Therefore, the difference between dipolar and radial mode damping rates may be assessed without explicit calculation of nonadiabatic effects in these layers.

Proceeding a similar way as in derivation of the variational expression for real eigenvalues, we begin with multiplying Eq. (1) by $\xi^{*}$ and integrating over the envelope. In further transformation, we make use of the Poisson and continuity equations and finally arrive at

$$
\begin{aligned}
\omega^{2} I= & -\int_{\Sigma_{b}}\left[\xi_{r}^{*}\left(p^{\prime}+\rho \Phi^{\prime}\right)+\frac{\Phi^{\prime}}{4 \pi G} \frac{\partial \Phi^{\prime} *}{\partial r}\right] \mathrm{d} \Sigma \\
& +\int_{V}\left(\frac{\left|p^{\prime}\right|^{2}}{p \Gamma_{1}}+A g \rho \frac{\left|\xi_{r}\right|^{2}}{r}-\frac{\left|\nabla \Phi^{\prime}\right|^{2}}{4 \pi G}\right) \mathrm{d}^{3} \boldsymbol{x}-E,
\end{aligned}
$$

where $E$ is the ad hoc added $\ell$-independent term describing the near surface effects that may contribute to damping (or driving).

Assuming $\left|\omega_{I}\right| \ll \omega_{R}$, we get from the imaginary part of Eq. (31)

$\omega_{I}=-\frac{W+E_{I}}{2 \omega_{R} I}$,

where

$W=\int_{\Sigma_{b}} \mathfrak{J}\left[\xi_{r}^{*}\left(p^{\prime}+\rho \Phi^{\prime}\right)+\frac{\Phi^{\prime}}{4 \pi G} \frac{\partial \Phi^{\prime} *}{\partial r}\right] \mathrm{d} \Sigma$,

which in terms of the radial eigenfunctions defined in Eqs. (2) and (3) becomes

$W=\left(\rho r^{5}\right)_{b}\left[\mathfrak{J}\left(\omega^{2} y_{1}^{*} y_{2}\right)+\frac{g}{r U} \mathfrak{J}\left(y_{3} y_{4}^{*}\right)\right]_{b}$.

With the use of the asymptotic solution given in Eq. (24), a more revealing expression for the damping rate caused by the wave losses may be obtained. Neglecting terms of the orders of $B$ and $\omega_{I} / \omega_{R}$ in comparison with 1 , we get

$W=\left(\rho r^{5}\right)_{b}\left[2 \omega_{R}^{2} \mathfrak{J}\left(\mathcal{Z}_{1}^{*} \mathcal{Z}_{2}\right)\left|\tilde{C}_{r}\right|^{2}+\frac{6 \omega_{I} \omega_{R}}{U}\left|\tilde{C}_{s}\right|^{2}\right]$. 
The leading terms in the coefficient at the $\tilde{C}_{s} \tilde{C}_{r}$ product cancel out. From Eqs. (14) and (16), we have

$\mathfrak{J}\left(\mathcal{Z}_{1}^{*} \mathcal{Z}_{2}\right)=0.842 \beta$

Thus, the total damping rate, $\gamma=-\omega_{I}$, is given by

$$
\gamma=\gamma_{W}+\gamma_{E}=-\omega_{I}=\frac{1}{I+I_{\mathrm{c}}}\left(0.842 \omega_{R}\left|\tilde{C}_{r}\right|^{2} \beta\left(\rho r^{5}\right)_{b}+\frac{E_{I}}{2 \omega_{R}}\right) .
$$

The expression for $I_{\mathrm{c}}$, which is the part of mode inertia arising in the core, is given in Eq. (28). The rapid component contributes only to the energy loss because the reflected wave was neglected.

The near surface effects have some influence on the eigenfunctions at the bottom of the envelope and, hence, on the value of $W$. This influence was assessed by adding to the coefficent at $y_{1}$ in the r.h.s. of Eq. (25) an imaginary number leading to the values of $\gamma_{E}$ of the order of those found by Dupret et al. (2009). The changes in values of $\gamma_{w}$ were found small enough to regard the two dominant sources of damping as additive.

Damping due to wave losses sharply decreases with increasing red giant luminosity. This is shown in the bottom panels of Figs. 6 and 7 for the dipolar modes with frequencies closest to $v_{\max }$ and for $p_{1,1}$ modes, respectively. The plots depict, for a number of envelope models, the values of $\tau_{W}=\gamma_{W}^{-1}$, that is, lifetimes limited only by the wave emission. There is some uncertainty in evaluated lifetimes following from the uncertainty in the derivative $\mathrm{d} A / \mathrm{d} r$, at the top of radiative interior. This derivative enters the inner boundary condition (see Eq. (24)) through parameter $\beta$ defined in Eq. (21). Our standard approach is to evaluate $\omega$ and $\tau_{W}$ assuming constant element abundance but the He abundance may rise immediately beneath the bottom of the convective envelope. To check consequences of this uncertainty, calculations were performed with the derivative increased by factor 10 . As expected, the effect on frequencies was negligible. The lifetimes became by factor about two longer. This is not much for such a drastic modification of the $A$ derivative and, especially, when the effect is compared with the four orders of magnitude increase of $\tau_{W}$ between $\log L=2$ and 3 . The general pattern of the $\tau_{W}(L)$ dependence, which we see in these two figures, are similar. The lifetimes are longer for the $p_{1,1}$ modes because at lower frequency the evanescent zone is wider, hence trapping is more efficient, and the wave amplitudes are lower. Decreasing mass at a specified luminosity acts in the same direction.

Let us now focus on modes with frequencies $v_{\max }$ which are of interest for comparison with observations. The lifetime due to wave losses increases rapidly with $L$ and it scales roughly as $M / L$. The average exponent, $w$, in the $\tau_{W} \sim L^{w}$ relation is close to 4 . It is interesting to compare the role of wave losses and the near surface damping in limiting mode lifetimes. For the latter effect we use values of $\tau_{E}$ calculated for radial modes of by Dupret et al. (2009). They calculated also lifetimes for nonradial modes but these depend on mode properties in the core. Therefore, it seems safer to rely on results radial modes at similar frequencies. For the trapped dipolar modes, which we want to consider, $I_{\mathrm{c}} / I$ is small and $I$ is close to mean value of the nearest radial modes. So that, we may use mean of the lifetimes of these modes as a crude assessment of $\tau_{E}$ for trapped dipolar modes.

In models with $M_{0}=2 M_{\odot}$ at $\log L=1.8$ and 2.1 , the values of $v_{\max }$ are 49 and $21 \mu \mathrm{Hz}$, respectively. From our Fig. 6, we find the values 20 and $110 \mathrm{~d}$ for $\tau_{W}$. The corresponding values of $\tau_{E}$ inferred from Figs. 7 and 8 of Dupret et al. are 20 and $30 \mathrm{~d}$. Thus, we may conclude that at $M_{0}=2 M_{\odot}$ and $\log L \gtrsim 2$ the wave losses become a secondary source of damping and become negligible at somewhat higher luminosity. At lower initial mass the same happens at accordingly lower luminosity. At $M_{0}=2 M_{\odot}$ and $\log L=2.1$ the total relative damping rate, $-\omega_{I} / \omega_{R}=\left(v_{\max } \tau\right)^{-1}=0.024$.

With this estimate, we may return to the matter of validity of the running wave approximation, discussed in Sect. 2. In order to use condition given in Eq. (18), we still need the values of $\Psi_{R}$ and $\int D \mathrm{~d} \Psi_{R}$ for which complete stellar models are needed. For the present estimate we will use numbers for the $M=2 M_{\odot}$ models calculated for Dziembowski et al. (2001) paper which should be adequate for this aim. The values of $\Psi_{R}$ and $\int D \mathrm{~d} \Psi_{R}$ at $L=2$ are $1.9 \times 10^{3}$ and 0.49 , respectively, while at $L=2.5$ the corresponding values are $1.0 \times 10^{4}$ and 370 (in this case the required exact expression for $k_{r}$ was used). The value of $\int D \mathrm{~d} \Psi_{R}$ grows much more rapidly with $L$ than $\Psi_{R}$ and it is clear that at the value slightly exceeding 2 the running wave approximation is justified even if only the linear radiative damping is considered.

\section{Discussion}

Solar-like oscillations in low luminosity red giants are interpreted as global mixed modes with both the gravity and acoustic cavities contributing, in various proportions, to the total inertia. At higher luminosity, a more adequate picture is that of acoustic modes of the convective envelope loosing energy via gravity wave emission from its bottom. With growing luminosity this loss becomes insignificant.

Transition between these two pictures is continuous but very sharp. From Dupret et al. (2009) and the arguments presented in this paper it follows that for stars of the initial mass $M_{0}=2 M_{\odot}$ the transition occurs near luminosity $L \gtrsim 100$, which corresponds (see Table 1) to $v_{\max }=27.5$ and $\Delta v=3.1 \mu$. At lower mass, the transition takes place at lower luminosity but the values of $\Delta v$ at the transition should be similar. In Fig. 3 of Mosser et al. (2010) most of data points lie above $\Delta v=3 \mu \mathrm{Hz}$ but there are also lots of data points below $\Delta v=2 \mu \mathrm{Hz}$ which are most likely associated with luminous objects, where only acoustic envelope modes are expected. The most striking feature shown in this figure is the universal pattern of the relative positions of the dipolar and radial modes across the whole range of $\Delta v$ values from 1 to $8 \mu \mathrm{Hz}$. The dipolar modes are located between radial modes somewhat shifted off the mid point toward high frequency. This is consistent with what is seen in Fig. 5 for the envelope modes. We may also see that some spread of the position of such modes is expected. Similar situation is expected for the mixed dipolar modes corresponding to minima of the inertia (Montálban et al. 2010).

A convincing evidence for the mixed mode excitation comes from detection of few dipolar modes near the mid points between consecutive radial modes (Beck et al. 2011; Bedding et al. 2011; Mosser et al. 2011). In the first two papers, three objects from the Kepler catalog are discussed. The values of $\Delta v$ are in the range of 7.9 to $10 \mu \mathrm{Hz}$ where stochastic excitation of mixed modes is indeed expected. However, Mosser et al. (2011) in their analysis of the CoRoT data find a large number of such objects in a wide range of $\Delta v$ extending down to $2 \mu \mathrm{Hz}$, that is well below the range expected range of mixed modes. They also report an evidence for mixed $\ell=2$ mode excitation and note this is in conflict with the Dupret et al. (2009) prediction that such modes should be eliminated by strong damping in the core. A possible solution of these two problems could be the gravity 
wave reflection caused by a steep composition gradient above the hydrogen burning shell, where most of the damping takes place. The large values of $\Delta T_{\mathrm{obs}}$ determined by Mosser et al. for the dipolar mixed modes at low $\Delta v$ are consistent with such an interpretation.

Acoustic envelope modes are not affected by what happens below the convective envelope. Their frequencies yield independent but similar information to radial modes because in both cases, frequencies are determined by sound speed distribution within the envelope. Only mixed modes probe the radiative interiors of red giants. Data on multiple peaks has been already used to distinguish between helium burning and other red giants (Bedding et al. 2011; Mosser et al. 2011). There is a prospect for going beyond that. In particular, we may get interesting constraints on element distribution from frequencies and on dissipative processes in the core from amplitudes and lifetimes. A new exciting result based on mixed dipolar modes is the evidence for fast rotation in red giant cores (Beck et al. 2012).

We do not yet have data satellite on oscillation in most luminous red giants $(\log L \gtrsim 2.5, \Delta v \lesssim 1)$. Rich data on such objects located in the Magellanic Clouds are available from OGLE project (Soszyński et al. 2009). The advantage of these data is that luminosity of individual objects is known to a good accuracy. The peaks occur at frequencies below $1 \mu \mathrm{Hz}$, which is consistent with prediction based on Eq. (26). The frequencies are in the range of lowest order p-modes. In a number of cases two clearly dominant peaks are seen. However, the frequency separations are by some $20 \%$ lower than expected large separation. This could not be explained in terms of two $\ell=0$ modes, nor in terms of the $\ell=0,1$ pair (Dziembowski \& Soszyński 2010). In the light of the discussion in the present paper the second option would appear more plausible but, in fact, it leads to a greater discrepancy. Satellite data on these objects might provide a key to the solution of this intriguing problem.
Acknowledgements. I am grateful to Masao Takata for reading a preliminary version of this paper and suggesting a number of corrections. This work was partially supported by the Polish MNiSW grant number N N203 379636.

\section{References}

Abramovitz, M., \& Stegun, I. A. 1972, Handbook of Mathematcal Functions (New York: John Wiley and Sons)

Aerts, C., Christensen-Dalsgaard, J., \& Kurtz, D. W 2010, Asteroseismology (Heidelberg: Springer)

Beck, P. G., Bedding, T. R., Mosser, B., et al. 2011, Science, 332, 205

Beck, P. G., Montalbán, J., Kallinger, T., et al. 2012, Nature, 481, 55

Bedding, T. R., \& Kjeldsen, H. 2003, PASA, 20, 203

Bedding, T. R., Mosser, B., Huber, D., et al. 2011, Nature, 471, 606

Christensen-Dalsgaard, J. 2004, Sol. Phys., 220, 137

Christensen-Dalsgaard, J. 2011, Asteroseismology, Canary Islands Winter School of Astrophysics, Vol. XXII, ed. P. L. Pallé (Cambridge University Press), in press [arXiv: 1106.5946v1]

Christensen-Dalsgaard, J., \& Gough, D. O. 2001, MNRAS, 326, 1115

Dupret, M. A., Belkacem, K., Samadi, R., et al. 2009, A\&A, 506, 57

Dziembowski, W. 1971, Acta Astron., 21, 289

Dziembowski, W. 1977, Acta Astron., 27, 95

Dziembowski, W. A., \& Soszyński, I. 2010, A\&A, 524, A88

Dziembowski, W. A., Gough, D. O., Houdek, G., \& Sienkiewicz, R. 2001, MNRAS, 328, 601

Hekker, S., Gilliland, R. L., Elsworth, Y., et al. 2011, MNRAS, 414, 2594

Kjeldsen, H., \& Bedding, T. R. 1995, A\&A, 293, 87

Kumar, P., \& Goodman, J. 1996, ApJ, 466, 946

Montalbán, J., Miglio, A., Noels, A. Scuflaire, R., \& Ventura, P. 2010, ApJ, 721, L182

Mosser, B., Belkacem, K., Goupil, M.-J., et al. 2010, A\&A, 517, A22

Mosser, B., Barban, C., \& Montalbán, J. 2011, A\&A, 532, A86

Osaki, Y. 1977, Pub. Astron. Soc. Japan, 29, 234

Pietrinferni, A., Cassisi, S., Salaris, M., \& Castelli, F. 2006, ApJ, 642, 797

Soszyński, I., Dziembowski, W. A., Udalski, A., et al. 2007, Acta Astron., 57, 1

Takata, M. 2005, Pub. Astron. Soc. Japan, 57, 275

Takata, M. 2006a, Pub. Astron. Soc. Japan, 58, 759

Takata, M. 2006b, Pub. Astron. Soc. Japan, 58, 893 